\title{
Bone marrow plasticity revisited: protection or differentiation in the kidney tubule?
}

\author{
Diane Krause ${ }^{1}$ and Lloyd G. Cantley ${ }^{2}$ \\ ${ }^{1}$ Department of Laboratory Medicine and 2Department of Internal Medicine, Yale University, New Haven, Connecticut, USA.
}

\begin{abstract}
Epithelial organs such as the intestine and skin have a relatively high rate of cell loss and thus require a reservoir of stem cells capable of both replacing the lost epithelia and maintaining the reservoir. Whether the kidney has such a stem cell niche has been a subject of great interest; the majority of data suggest that replacement of renal epithelial cells occurs via dedifferentiation and proliferation of existing tubular cells, while some studies demonstrate the presence of potential tubular stem cells in the renal interstitium. However, recent reports have suggested that the bone marrow may also be a source of stem cells for tubule turnover and/or repair. In this issue of the JCI, 2 groups explore the role of endogenous cells versus bone marrow-derived cells in mediating tubule repair. Duffield and colleagues demonstrate that bone marrow does contain cells capable of protecting the kidney from ischemic injury, but found that these cells do not act by direct incorporation into the repaired tubular segments (see the related article beginning on page 1743). In contrast, Lin and coworkers found that some bone marrow-derived cells do appear to incorporate into the injured tubule as epithelial cells (see the related article beginning on page 1756). Importantly, both groups conclude that the majority of tubule repair occurs via proliferation of endogenous renal cells rather than incorporation of bone marrow-derived cells.
\end{abstract}

\section{Cells derived from bone marrow may functionally participate in renal repair}

In both humans and mice, data has been obtained in support of the ability of cells emanating from the bone marrow to become renal epithelial cells. Several groups have reported the presence of Y chromosome-positive renal tubular cells in kidneys of male patients who received a renal transplant from a female recipient, which suggests that cells from outside the kidney can populate the renal tubule $(1,2)$. The percentage of Y chromosome-positive renal tubule cells ranged from $0.6-6.8 \%$ in one study (1) and was about $1 \%$ in the other (2). In female mice that had undergone bone marrow transplantation from male donors, Y chromosome-positive tubule cells that expressed epithelial markers were reported in one study to be present at low levels in $7.9 \%$ of the renal tubules (1) and in a different study to comprise up to $8 \%$ of renal

Nonstandard abbreviations used: BMSC, bone marrow-derived stem cell; BUN, blood urea nitrogen; I/R, ischemia/reperfusion; MSC, marrow stromal cell.

Conflict of interest: The authors have declared that no conflict of interest exists.

Citation for this article: J. Clin. Invest. 115:1705-1708 (2005). doi:10.1172/JCI25540. tubule cells (3). Following transplantation of whole bone marrow from $\beta$-gal transgenic Rosa mice into wild-type recipients, 2 studies (including one by our group) reported that $3-6 \%$ of renal tubules in the cortex and up to $20 \%$ in the medulla contained at least 1 cell that stained positive with X-gal 1 week after ischemic renal injury $(3,4)$. As $\beta$-gal-positive cells were not seen in kidneys that had been subjected to ischemia/reperfusion (I/R) in the absence of bone marrow transplantation, the authors of both studies interpreted this result as suggestive of a major role of the bone marrow in tubular epithelial cell repair. Our results further demonstrated that infusion of lineage-negative bone marrow cells could partially reverse the exaggerated rise in blood urea nitrogen (BUN) seen in mice that had been subjected to lethal irradiation followed by I/R (4), which suggests that these cells can play a functional role in the recovery process.

The lineage-negative fraction of bone marrow contains multiple cell types, including rare subpopulations of HSCs and marrow stromal cells (MSCs, alternatively referred to as mesenchymal stem cells). Particular attention has been focused in recent years on the potential of bone marrow-derived stem cells (BMSCs) to differentiate into and/or fuse with other cell types and thereby functionally incorporate into nonhematopoietic organs such as liver and lung (refs. 5, 6; reviewed in ref. 7). Morigi and coworkers found that the MSC population of lineage-negative bone marrow cells has the primary capacity to participate in renal tubule repair, whereas the HSC population mediates a lesser protective effect (8). They demonstrated that infusion of large numbers of male MSCs markedly diminished the initial rise in BUN in the setting of cisplatin-induced tubular injury in female recipients and found $\mathrm{Y}$ chromosome-positive tubular cells in the kidneys of these animals, although the percentage of MSC-derived tubule cells was not stated. Similar findings were reported by Herrera and colleagues following transplantation of GFP-expressing MSCs into mice that were subjected to glycerol-induced tubular injury (9). In their study, $22 \%$ of tubular cells were found to be GFP-positive after injury.

Is the most important role of the bone marrow-derived cell protective rather than reparative in the kidney? The study by Duffield et al. reported in this issue of the JCI (10) demonstrates the ability of purified MSCs to improve renal function in an I/R model of acute tubular injury in the mouse, but the authors noted an important difference from the prior studies. They found that the effects of transplanted MSCs were independent of any direct contribution to the renal parenchyma, either tubular or endothelial in nature. Furthermore, using 3 separate approaches for tracking donorderived cells (GFP, Y chromosome, and $\beta$-gal), they found that animals undergoing whole bone marrow transplantation followed by acute renal injury also failed to exhibit any tubular cells derived from donor bone marrow, although they did find small numbers of cells expressing endothelial markers that were of bone marrow origin in the kidneys of these animals (Figure 1). The absence of bone marrow-derived endothelial cells in kidneys from mice transplanted with purified MSCs suggests that the endothelial cells come from a non-MSC fraction of the bone marrow. 


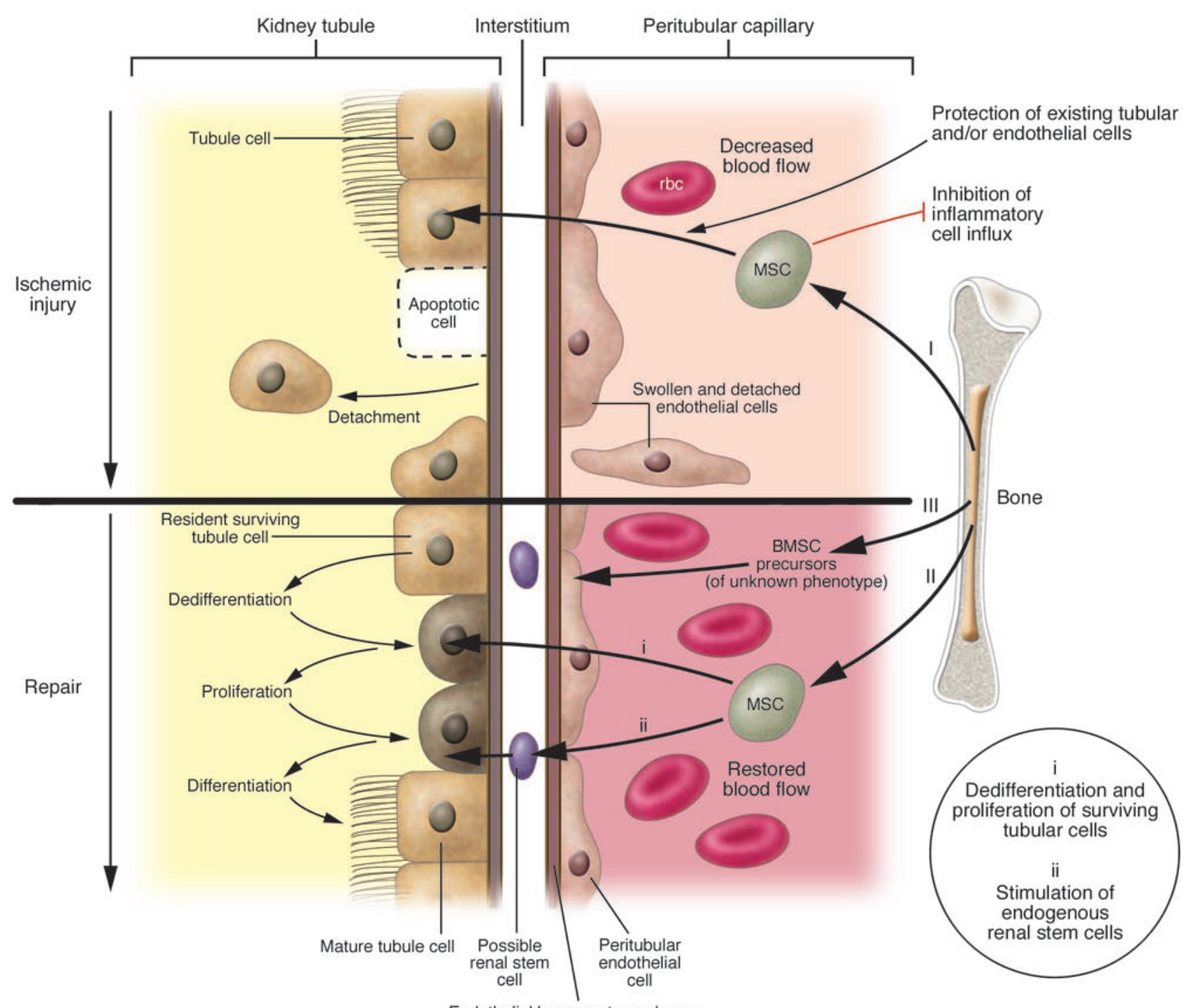

Figure 1

Potential roles of bone marrow-derived cells in renal protection/repair. After ischemic injury, the brush border of proximal tubular cells is rapidly lost, followed by either dedifferentiation and proliferation of these cells or cell detachment and death due to necrosis or apoptosis. The observation that the initial decline in renal function following ischemic injury is markedly diminished in animals receiving large numbers of MSCs suggests that these cells act to prevent the acute phase of the injury either by directly inhibiting cell death and/or by preventing inflammatory cell influx (I). Once injury has occurred, repair of the tubule requires the dedifferentiation, migration, and proliferation of the surviving tubular cells. In addition, it has been proposed that resident renal stem cells participate in the reparative phase by migrating into the tubule and differentiating into epithelial cells (14). MSCs may direct this phase of tubule repair by secreting a factor (or factors) that promotes tubular cell dedifferentiation and proliferation or by stimulating the influx of resident stem cells (II). A second bone marrow cell type, possibly HSCs, appears to serve as a reservoir of endothelial progenitor cells, which potentially promotes endothelial repair and increases renal medullary blood flow (III). Finally, the possibility remains that bone marrow-derived cells can, very rarely, undergo differentiation into or fuse with existing tubular cells to directly participate in tubule repair. The infrequent occurrence of these last 2 processes suggests that they are not critical for functional recovery from acute ischemic renal injury.

The idea that MSCs may protect the renal tubule without directly participating in tubule repair is supported by recent work by Togel and coworkers (11). Using fluorescently labeled MSCs, they found that these cells protected the kidney from ischemic injury but were detectable in the renal microvasculature for only $1-2$ days after infusion and were not observed to differentiate into tubular or endothelial cells during the first 3 days. In contrast, the present study by Lin and coworkers (12) shows that infusion of whole bone marrow (that would be expected to contain very few MSCs) failed to prevent ischemic renal injury and raises the argument that protection may require the infusion (or mobilization) of large numbers of MSCs.
Duffield and colleagues (10) suggest that the $\beta$-gal-positive tubular cells reported in prior studies may have been detected as a result of increased intrinsic $\beta$-gal activity in the injured tubule rather than the presence of $\beta$-gal-positive bone marrow cells. They also suggest that the $\mathrm{Y}$ chromosome-positive tubular cells detected in previous studies of female mice following transplanta- 
tion of bone marrow from male donor mice are likely artifacts of imaging caused by the staining technique or the superimposition of a tubular cell and an infiltrating bone marrow-derived cell.

\section{How can we use bone marrow- derived cells to improve renal function?}

The work by these 2 groups raises several interesting and important questions. First, why is the degree to which bone marrow-derived cells differentiate into renal epithelial cells so different in the different studies? Are the previous reports incorrect, as suggested by the Duffield et al. study (10), or is differentiation of bone marrowderived cells and/or their fusion with resident tubular cells an uncommon but real occurrence that is dependent upon distinct experimental conditions, as supported by the present study by Lin et al. (12)?

Based on our recent studies using transplantation of male bone marrow into female recipients, we agree with Duffield and colleagues (10) that the prior $\beta$-gal studies markedly over-represented the contribution of bone marrow-derived cells to the repopulation of ischemically injured tubules during the repair process, although our reasoning behind this conclusion is somewhat different from that of Duffield et al. In our work, significant numbers of $\beta$-gal-positive tubular cells were only seen in the kidneys of animals that had received a bone marrow transplant from a Rosa donor mouse and only when those kidneys had been subjected to I/R (4). However, careful review of those kidney sections revealed no $\beta$-gal-positive interstitial cells, even though these cells should be clearly detectable. Thus, we presently believe that the $\beta$-gal-positive bone marrow cells that have infiltrated the interstitium in response to injury may leak the enzyme and/or product, which is apparently then sequestered in the nearby injured tubular cells. It remains unclear whether this transfer of the $\beta$-gal enzyme or the dye occurs due to a physiologically relevant communication between the 2 cell types or is an artifact of fixation or staining. The alternative possibility that $\beta$-gal present in the circulation of these chimeric animals is filtered and taken up by the proximal tubule cells appears less likely, due to the large molecular weight of $\beta$-gal (a homotetramer with predicted molecular weight of $540 \mathrm{kDa}$ ) and the fact that we failed to see these intensely stained tubular cells in the contralateral kidney in experi- ments in which only 1 kidney had been ischemically injured (4).

Ultimately, regardless of the exact mechanism for the increased detection of $\beta$-gal previously reported by us (4) as well as by Lin and coworkers (3), the current studies by Duffield et al. (10) and Lin et al. (12) suggest that the $\beta$-gal model is unreliable for use in these studies and has led to a significant overestimation of the role of bone marrow-derived cells in directly repairing injured tubules. This conclusion, however, does not resolve the question of whether bone marrow cells ever contribute directly to tubule regeneration. The results of the current study by Lin and colleagues (12) are at odds with those of Duffield et al. (10) in that they continue to support this possibility by demonstrating the presence of Y chromosome-positive, CD45-negative cells in the renal tubules of female mice subjected to I/R injury and male whole bone marrow transplantation. These cells were located primarily in the proximal tubule and expressed the proximal tubule marker Lotus tetragonolobus agglutinin (LTA), which supports the possibility that they had differentiated into or fused with renal tubular cells. However, when Lin et al. transplanted bone marrow from a transgenic mouse that expresses enhanced GFP uniquely in renal epithelium, no GFP-positive tubule cells were found in the recipient mice after ischemic injury. While a negative result using this approach could be due to silencing of the transgene in the bone marrow-derived cells, it adds to the concerns that positive results reported in other transplant models might be due to misinterpretation or artifact. Importantly, in agreement with the study by Duffield et al., Lin and colleagues demonstrate that the majority of the cells that were dividing to repair the injured tubules came from an endogenous cell population, rather than from bone marrow-derived cells.

Thus, despite reports by multiple investigators of renal tubular cells that are bone marrow-derived (1-4, 8, 9), the present studies by Duffield et al. (10) and Lin et al. (12) demonstrate that in vivo differentiation of BMSCs into renal tubular cells may not occur at all, or is at most a minor component of the repair process after ischemic injury. It remains to be seen whether recent studies demonstrating significant numbers of renal tubular cells derived from MSCs following toxic tubular injury $(8,9)$ will be confirmed when subjected to 3-dimensional imaging with deconvolution. In our opinion, conclusive proof that bone marrow-derived cells can differentiate into renal tubular cells in vivo will be provided only when these cells are isolated and characterized at the phenotypic, genetic, and functional levels.

This leads to the second, and more clinically important, question. If bone marrow-derived cells do not participate in renal tubule repair by directly differentiating into large numbers of tubular cells, what is the mechanism by which they protect the kidney from ischemic and/or toxic injury? The observation in the Duffield et al. study that bone marrow-derived cells can differentiate into or fuse with endothelial cells (10) suggests that augmentation of endothelial repair in the vasa recta could be responsible for the improved renal function following MSC infusion in mice subjected to I/R. In support of this possibility, Duffield et al. demonstrate that culture of the MSCs under conditions that promote in vitro differentiation into endothelial cells was required for their protective effect in vivo. However, even under these conditions, they failed to detect donor MSCs in the kidney following I/R, either in the tubules or the endothelium. This result suggests that even though some fraction of bone marrow-derived cells may be capable of becoming vascular endothelial cells in the ischemically injured kidney, this process is unlikely to be important for the protective effect provided by the MSCs.

Our ability to determine the exact mechanism by which MSCs protect the kidney is presently hampered by our poor understanding of their identity. MSCs are typically characterized as nonhematopoietic bone marrow-derived cells that adhere to and proliferate on tissue culture dishes and thus may or may not represent identical cell populations when derived in different laboratories (13). Defining a surface phenotype that allows purification of these cells and determining which subpopulation of MSCs actually possesses the ability to protect the kidney from injury will be important for understanding the mechanism(s) by which they protect ischemic tissues. However, because the surface membrane protein expression is dynamic on primary cells in culture, defining a functional phenotype would be optimal.

The present results by Duffield et al. (10) are most consistent with the idea that MSCs, when cultured under the proper conditions and given in sufficient numbers, secrete a factor (or factors) that protects the existing tubular cells from apoptosis, promotes their proliferation, suppresses the inflammatory response after reperfusion, or augments the influx and differentiation of endogenous 
renal stem cells (Figure 1). The failure of whole bone marrow to have this protective effect (12) may be due to the extremely small numbers of MSCs present in the bone marrow or the large numbers of inflammatory cells infused in this preparation. It remains unclear whether the protective effect of MSCs requires them to leave the bone marrow and transit through the renal circulation or whether these cells can exert protective effects from distant sites. Identifying the protective factor(s) and the signals that prompt MSCs to secrete it should now be a priority in our attempts to develop new therapeutic approaches for improving patient outcomes following acute renal failure.

Address correspondence to: Lloyd G. Cantley, Department of Internal Medicine, Yale University, 333 Cedar Street, Box 208029, New Haven, Connecticut 06520,
USA. Phone: (203) 785-7110; Fax: (203) 785-3756; E-mail: Lloyd.Cantley@yale.edu.

1. Poulsom, R., et al. 2001. Bone marrow contributes to renal parenchymal turnover and regeneration. J. Pathol. 195:229-235.

2. Gupta, S., Verfaillie, C., Chmielewski, D., Kim, Y., and Rosenberg, M.E. 2002. A role for extrarenal cells in the regeneration following acute renal failure. Kidney Int. 62:1285-1290.

3. Lin, F., et al. 2003. Hematopoietic stem cells contribute to the regeneration of renal tubules after renal ischemia-reperfusion injury in mice. J. Am. Soc. Nephrol. 14:1188-1199.

4. Kale, S., et al. 2003. Bone marrow stem cells contribute to repair of the ischemically injured renal tubule. J. Clin. Invest. 112:42-49. doi:10.1172/ JCI200317856.

5. Lagasse, E., et al. 2000. Purified hematopoietic stem cells can differentiate into hepatocytes in vivo. Nat. Med. 6:1229-1234.

6. Krause, D.S., et al. 2001. Multi-organ, multi-lineage engraftment by a single bone marrow-derived stem cell. Cell. 105:369-377.

7. Herzog, E.L., Chai, L., and Krause, D.S. 2003. Plasticity of marrow-derived stem cells [review]. Blood. 102:3483-3493.
8. Morigi, M., et al. 2004. Mesenchymal stem cells are renotropic, helping to repair the kidney and improve function in acute renal failure. J. Am. Soc. Nephrol. 15:1794-1804.

9. Herrera, M.B., et al. 2004. Mesenchymal stem cells contribute to the renal repair of acute tubular epithelial injury. Int. J. Mol. Med. 14:1035-1041.

10. Duffield, J. 2005. Restoration of tubular epithelial cells during repair of the postischemic kidney occurs independently of bone marrow-derived stem cells. J. Clin. Invest. 115:1743-1755. doi:10.1172/JCI22593.

11. Togel, F., et al. 2005. Administered mesenchymal stem cells protect against ischemic acute renal failure through differentiation-independent mechanisms. Am. J. Physiol. Renal Physiol. doi:10.1152/ ajprenal.00007.2005.

12. Lin, F., Moran, A., and Igarashi, P. 2005. Intrarenal cells, not bone marrow-derived cells, are the major source for regeneration in postischemic kidney. J. Clin. Invest. 115:1756-1764. doi:10.1172/JCI23015.

13. Javazon, E.H., Beggs, K.J., and Flake, A.W. 2004. Mesenchymal stem cells: paradoxes of passaging. Exp. Hematol. 32:414-425.

14. Oliver, J.A., Maarouf, O., Cheema, F.H., Martens, T.P., and Al-Awqati, Q. 2004. The renal papilla is a niche for adult kidney stem cells. J. Clin. Invest. 114:795-804. doi:10.1172/JCI200420921.

\title{
Harlequin ichthyosis unmasked: a defect of lipid transport
}

\author{
Alain Hovnanian \\ Department of Medical Genetics and INSERM U563, Purpan Hospital, Toulouse, France.
}

\begin{abstract}
Harlequin ichthyosis (HI) - the most severe form of keratinizing disorders, often lethal in the neonatal period - is characterized by a profound thickening of the keratin skin layer, a dense "armor"-like scale that covers the body, and contraction abnormalities of the eyes, ears, and mouth. In this issue of the JCI, Akiyama et al. report that mutations in ABCA12 caused defective lipid transport that significantly impacted normal development of the skin barrier (see the related article beginning on page 1777). Lipid secretion was recovered after corrective $A B C A 12$ gene transfer into patient keratinocytes. These results should allow for early prenatal diagnosis of $\mathrm{HI}$ and lend hope to the possibility of a specific treatment for this devastating disorder.
\end{abstract}

Waring pointed to what is believed to be the first harlequin fetus described in the US in the diary of Reverend Oliver Hart in 1750 (1). Harlequin ichthyosis (HI) is believed to be inherited in an autosomalrecessive manner, and affected newborn infants are encased in "armor"-like thick, yellow plates of scales with deep red fissuring. The skin is pulled tight such that the face loses its normal appearance and

Nonstandard abbreviations used: HI, harlequin ichthyosis.

Conflict of interest: The author has declared that no conflict of interest exists.

Citation for this article: J. Clin. Invest. 115:1708-1710 (2005). doi:10.1172/JCI25736. appears frog-like, with eversion of the eyelids (ectropion) and lips (eclabion) and flattening of the ears and nose. The extremities are swollen due to constriction by massive thickening of the skin. Liveborn infants usually die within the first days of life from respiratory, infectious, and/or dehydration-related complications. Some patients treated with retinoids, synthetic derivatives of vitamin A, have survived and subsequently develop severe ichthyosis. The cause of HI, however, has remained elusive, and late prenatal diagnosis has until now relied on electron microscopic examination of tissue sampled by invasive fetal skin biopsy.

\section{Lipid transport: a likely suspect}

Members of the ABCA subclass of the large $A B C$ transporter protein family bind ATP for the active transport of lipids across cell membranes against a concentration gradient. $A B C A 1$ has been shown to be the causative gene in Tangier disease, a disorder of cholesterol transport between liver and other tissues (2-5), while mutations in $A B C A 4$ (expressed exclusively in photoreceptors of the eye for the transport of retinol) cause Stargardt disease, recessive retinitis pigmentosa, or cone-rod dystrophy, in which the abnormal accumulation of retinoids results in the development of macular dystrophy and loss of central vision (6-8).

Lipid processing in the skin is essential for the protective function of the stratum corneum, the most external layer of the epidermis (9). Corneocytes, attached to each other by corneodesmosomes and embedded in intercellular lipid lamellae, form a cornified layer that acts as a barrier between the internal and external environment for bodily defense. The lipid lamellae are derived from lamellar granules, the 